Ann. Génét. Sél. anim., I975, 7 (I), 13.22.

\title{
ÉTUDE D'UN GÈNE DE NANISME LIÉ AU SEXE CHEZ LA POULE : HEURE DE PONTE ET CARACTÉRISTIQUES DES CEUFS SUCCESSIFS DANS LA SÉRIE DE PONTE
}

\author{
M. AMIN-BAKHCHE et P. MÉRAT \\ Laboratoire de Génétique factorielle, \\ Centre national de Recherches zootechniques, I. N. R. A., \\ 78350 Jouy en Josas
}

\begin{abstract}
RÉSUMÉ
L'observation de l'heure de ponte approximative des œufs successifs de séries de diverses tailles a été faite, en début de ponte (mars I97I) puis après 4 à 5 mois de production (juin I97I) pour des poules naines $(d w)$ et de taille normale $(D w)$. Les œufs pondus isolément le sont plus tard dans la journée pour les naines. Ce n'est pas le cas pour le premier ouf des séries plus longues ; par contre, le décalage d'heure de ponte entre les oufs successifs de ces séries est plus important chez les poules $d w$, surtout en mars. Ce décalage est par ailleurs, au total, nettement plus grand en juin qu'en mars.

Le poids des œufs, celui des jaunes, la hauteur de l'albumen et l'épaisseur des coquilles ont été également mesurés, suivant la place dans la série, pour les mêmes poules $D w$ et $d w$ en mars et juin I97I, et pour d'autres en mars et juin 1972. Dans l'ensemble, le poids de l'œuf et celui du jaune diminuent davantage au cours de la série pour les poules naines, et l'épaisseur de la coquille semble augmenter un peu plus. Ceci confirme des observations antérieures.

Il existe, d'autre part, une différence moyenne d'épaisseur des coquilles à l'avantage des naines en début de ponte seulement, peut-être par suite de leur entrée en ponte un peu plus tardive.
\end{abstract}

\section{INTRODUCTION}

De nombreux auteurs, depuis HUTr (I959) ont mis en évidence la diminution du taux de ponte apportée par le gène de nanisme lié au sexe $d w$, du moins dans les souches de petite taille. JAAP et MoHammadian (I969) notent une vitellogenèse moins active chez les poules naines. Selon MÉRAT (I972), la longueur des séries de ponte (nombre d'œufs pondus à des jours successifs sans interruption) est plus petite pour les poules $d w$. Ces faits nous ont incités à comparer, pour les deux génotypes 
M. AMIN-BAKHCHE, P. MÉRAT

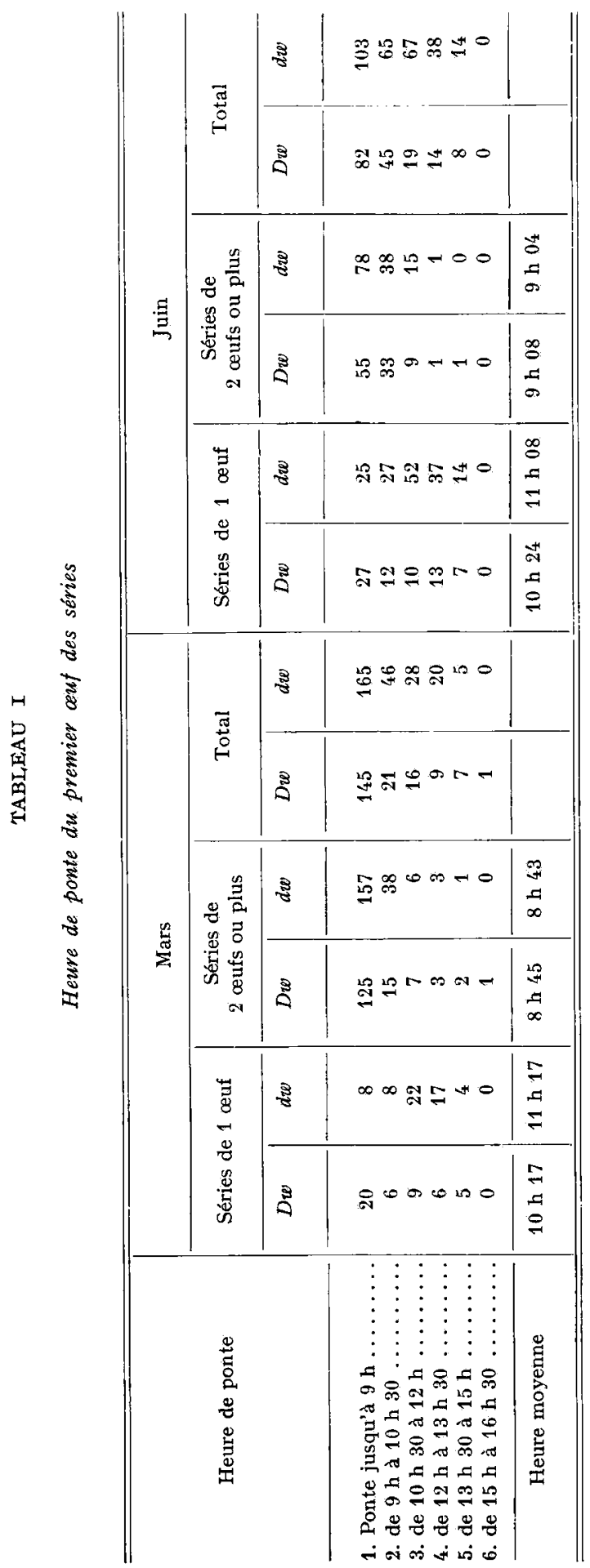


$D w$ et $d w$, 1'heure de ponte, qui peut refléter soit la durée de séjour de 1'œuf dans l'oviducte, soit le décalage dans le moment de l'ovulation au cours de la série. Nous avons, en outre, comparé l'évolution du poids et de certains constituants de l'œuf dans la série pour des poules des deux génotypes.

\section{MATÉRIEL ET MÉTHODES}

Les poules utilisées pour le relevé de l'heure de ponte, issues du troupeau expérimental de Jouy en Josas, étaient écloses en septembre 1970. Elles comprenaient 82 naines (dw) et 88 normales $(D w)$ issues de 6 familles de demi-sœurs, le père étant hétérozygote $D w d w$. Nos observations avaient lieu en cages individuelles en mars, puis fin juin 1971, c'est-à-dire en début de ponte, puis après 4 à 5 mois de production. L'heure de ponte des oufs de chaque poule était évaluée approximativement par 6 passages dans le poulailler, espacés d'une heure et demie, de 9 heures à $\mathrm{I} 6$ heures 30 inclus, chaque jour du $\mathrm{I}^{\mathrm{er}}$ au $\mathrm{I} 9$ mars, puis du 22 juin au 5 juillet. A ces passages correspondent 6 classes d'heure (tabl. I). L'importance des intervalles ne permettait donc de mettre en évidence que des différences assez grandes.

Quant à l'évolution du poids de l'œuf dans la série, elle était notée dans les séries où tous les œufs avaient pu être pesés (absence de cassures importantes) durant ces deux périodes.

Sur les mêmes données, l'épaisseur des coquilles était mesurée au cours de la série en mars, et les jaunes pesés sur un échantillon plus restreint en mars et juin. En outre, sur des poules $D w$ et $d w$ de même origine et de même âge, en 1972 , la variation du poids de l'œuf et celle de l'épaisseur de la coquille étaient enregistrées en mars et juin, le poids du jaune sur un échantillon limité en juin, sur une durée de 2 semaines chaque fois.

\section{RÉSULTATS}

\section{I. - Heure de ponte du premier ceuf des séries}

Nous présentons dans le tableau r l'heure de la ponte du premier œuf des séries suivant leur longueur. Les séries comportant plus d'un seul œuf ne paraissent pas différer sensiblement quant à l'heure de ponte de leur premier œuf et sont toutes regroupées.

Un $\chi^{2}$ de contingence pour les séries de I œuf (en regroupant en une seule les classes d'heures 2 et 3 d'une part, 4 à 6 de l'autre) et pour les séries de plus d'un œuf (avec la classe I - ponte jusqu'à 9 heures - comparée aux autres groupées) est présenté au tableau 2.

TABLEAU 2

$\chi^{2}$ de contingence entre poules naines et normales pour l'heure de ponte du 1 er ceuf des séries

\begin{tabular}{|c|c|c|c|c|}
\hline \multirow{3}{*}{ Taille de série } & \multicolumn{4}{|c|}{ Période } \\
\hline & \multicolumn{2}{|r|}{ Mars } & \multicolumn{2}{|r|}{ Juin } \\
\hline & d.l. & $\chi^{2}$ & d.l. & $\chi^{2}$ \\
\hline Séries de 1 œuf & 2 & $11,70(\mathrm{P}<0,01)$ & 2 & $14,60(\mathrm{P}<0,01)$ \\
\hline Séries de 2 œufs ou plus & 1 & 1,37 (NS) & 1 & 0,29 (NS) \\
\hline
\end{tabular}


Il n'était pas possible de faire une analyse de variance, étant donnée la dissymétrie accentuée de la distribution.

Pour les séries d'un seul œuf, les poules normales ont tendance à pondre plus tôt le matin que les naines. Pour les premières la fréquence maximum de ponte se situe avant 9 heures, pour les secondes entre Io $h 30$ et $\mathrm{I} 2$ heures. Par ailleurs, il y a peu de différence entre mars et juin pour chaque génotype.

Pour les plus longues séries, au contraire, l'heure de ponte du rer œuf ne diffère pas significativement pour les deux génotypes comparés, tant en mars qu'en juin, mais l'heure moyenne de ponte est dans l'ensemble plus tardive en juin.

\section{2. - Décalage de l'heure de ponte au cours de la série}

L'intervalle moyen en heures entre la ponte des œufs consécutifs d'une même série, par taille de série et pour chaque génotype, est donné dans le tableau 3 .

\section{TABLEAU 3}

Intervalle de temps moyen

entre la ponte de deux oufs consécutits d'une série

\begin{tabular}{|c|c|c|c|c|c|c|c|c|c|c|}
\hline \multirow{3}{*}{$\begin{array}{c}\text { Taille } \\
\text { des séries }\end{array}$} & \multicolumn{5}{|c|}{ Mars } & \multicolumn{5}{|c|}{ Juin } \\
\hline & \multicolumn{2}{|c|}{$D w$} & \multicolumn{2}{|c|}{$d w$} & \multirow{2}{*}{$\begin{array}{c}\text { Différence } \\
d w-D w\end{array}$} & \multicolumn{2}{|r|}{$D w$} & \multicolumn{2}{|c|}{$d w$} & \multirow{2}{*}{$\begin{array}{c}\text { Différence } \\
d w-D w\end{array}$} \\
\hline & $n$ & $\overline{\mathrm{X}}(1)$ & $n$ & $\overline{\mathrm{x}}$ & & $n$ & $\bar{x}$ & $n$ & $\overline{\mathrm{X}}$ & \\
\hline 2 & 28 & 26 h 11 & 65 & 27 h 27 & $1 \mathrm{~h} 16$ & 53 & 27 h 31 & 76 & $28 \mathrm{~h} 00$ & 0 h 29 \\
\hline 3 & 40 & $24 \mathrm{~h} 56$ & 94 & $26 \mathrm{~h} 36$ & 1 h 39 & 66 & 26 h 51 & 40 & $27 \mathrm{~h} 06$ & 0 h 15 \\
\hline 4 & 36 & $24 \mathrm{~h} 55$ & 75 & 25 h 59 & $1 \mathrm{~h} 04$ & 57 & 25 h 34 & 24 & $25 \mathrm{~h} 37$ & $0 \mathrm{~h} 03$ \\
\hline $\begin{array}{l}5 \\
\text { et plus }\end{array}$ & 128 & $24 \mathrm{~h} 22$ & 106 & $24 \mathrm{~h} 5 /$ & $0 \mathrm{~h} 32$ & 27 & 25 h 19 & 20 & 25 h 07 & -0 h 12 \\
\hline
\end{tabular}

$\boldsymbol{n}=$ nombre de couples d'œufs consécutifs d'une même série.

Les naines, en moyenne, pondent leurs œufs successifs à des intervalles de temps plus espacés que les poules normales, la différence étant de moins en moins marquée pour les plus longues séries (maximum dans les séries de 3 œufs en mars et celles de 2 œufs en juin). Pour le total, cette différence est de I heure 20 minutes en moyenne en mars, et seulement de 16 minutes en juin.

Une analyse de variance a été faite sur le caractère " différence moyenne d'heure de ponte entre œufs consécutifs d'une même série ", avec pour facteurs de variation contrôlés la taille de la série $(2,3,4,5$ ou plus) et le génotype au locus $D w$. L'analyse tenait compte des effectifs inégaux, et l'interaction était estimée de façon approchée (SNE DECOR et CoCHRAN, I969), suivant un modèle à effets fixés. Les conditions requises pour l'analyse n'étaient remplies que de façon approximative : nombre

(1) Exprimée en heures et minutes. 
limité de classes pour la variable de base, variance non égale à l'intérieur de chaque taille de série. D'autre part dans chaque sous-groupe à génotype et à taille de série fixés, les variables élémentaires n'étaient pas totalement indépendantes à cause d'un effet individuel possible commun aux séries d'une même poule, et il était exclu de pouvoir en tenir compte dans notre analyse. Enfin, les tailles de séries étaient assez inégalement réparties parmi les génotypes $D w$ et $d w$. Elles l'étaient encore plus aux deux dates, (mars et juin) et c'est pourquoi nous avons fait deux analyses séparées pour chacune. Nous présentons cependant cette analyse (tabl. 4), les effets principaux (séries en mars et juin, génotypes en mars) atteignant un niveau de signification très élevé. L'interaction, quant à elle, n'est en aucun cas significative.

TABLEAU 4

Analyse de variance sur la différence moyenne d'heure de ponte entre oufs consécutifs d'une même série

\begin{tabular}{|c|c|c|c|}
\hline Source de variation & d. 1 . & Carré moyen & $\mathrm{F}$ \\
\hline \multicolumn{4}{|c|}{ Mars } \\
\hline $\begin{array}{l}\text { Génotypes } \ldots \ldots \ldots \ldots \ldots \ldots \\
\text { Séries } \ldots \ldots \ldots \ldots \ldots \\
\text { Interaction } \ldots \ldots \ldots \ldots \ldots \ldots \\
\text { Résiduelle } \ldots \ldots \ldots \ldots \ldots \ldots\end{array}$ & $\begin{array}{r}1 \\
3 \\
3 \\
235\end{array}$ & $\begin{array}{r}27,20 \\
21,38 \\
1,11 \\
1,07\end{array}$ & $\begin{array}{l}25,46 * * * \\
20,01 * * * \\
1,03\end{array}$ \\
\hline \multicolumn{4}{|c|}{ Juin } \\
\hline $\begin{array}{l}\text { Génotypes } \ldots \ldots \ldots \ldots \ldots \ldots \\
\text { Séries } \ldots \ldots \ldots \ldots \ldots \ldots \\
\text { Interaction } \ldots \ldots \ldots \ldots \ldots \\
\text { Résiduelle } \ldots \ldots \ldots \ldots \ldots \ldots\end{array}$ & $\begin{array}{r}1 \\
3 \\
3 \\
212\end{array}$ & $\begin{array}{r}0,52 \\
21,86 \\
0,09 \\
0,70\end{array}$ & $\begin{array}{l}0,74 \\
31,27 * * * \\
0,13\end{array}$ \\
\hline
\end{tabular}

TABLEAU 5

Analyse de variance de la différence moyenne d'heure de ponte des aufs consécutifs par série avec les facteurs génotype, famille de père et longueur de série

\begin{tabular}{|c|c|c|c|}
\hline Source de variation & d.l. & C. M. & $\mathbf{F}$ \\
\hline 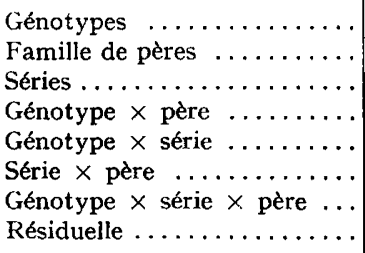 & $\begin{array}{r}1 \\
5 \\
1 \\
5 \\
1 \\
5 \\
5 \\
226\end{array}$ & $\begin{array}{r}21,308 \\
2,037 \\
35,646 \\
2,070 \\
0,871 \\
1,354 \\
1,604 \\
0,880\end{array}$ & $\begin{array}{c}24,213^{* * *} \\
2,314^{*} \\
40,506^{* * *} \\
2,352^{*} \\
0,989 \\
1,538 \\
1,822\end{array}$ \\
\hline
\end{tabular}

*** : Significatif au seuil 1 p. 1000.

* : Significatif au seuil 5 p. 100. 
Sur les données de mars, nous avons fait une autre analyse de variance avec les effets génotype, famille de même père et longueurs de série scindées en deux sousgroupes, l'un avec les séries de 2 et 3 œufs, l'autre avec les séries de 4 œufs ou plus. Cette analyse, faite de façon analogue à la précédente en ce qui concerne la prise en compte de l'inégalité des effectifs et l'estimation des interactions, figure au tableau 5 .

Le résultat confirme les conclusions précédentes, bien que l'interaction entre pères et génotypes atteigne le seuil $5 \mathrm{p}$. roo de signification et rende plus délicat à interpréter l'effet du génotype.

\section{3. - Variation du poids et des constituants de l'ouf au cours de la série}

Préalablement à l'examen de la variation des constituants de l'œuf au cours de la série, en mars et juin I97 I et 1972, le tableau 6 indique la valeur moyenne de ces constituants par génotype et par période.

\section{TABLEAU 6}

Valeur moyenne des constituants de l'auf par génotype et par période

\begin{tabular}{|c|c|c|c|c|c|c|c|c|c|c|}
\hline \multirow[b]{2}{*}{ Période } & \multicolumn{5}{|c|}{$D w$} & \multicolumn{5}{|c|}{$d w$} \\
\hline & $\begin{array}{l}\text { Pds de } \\
\text { l'ouf } \\
\text { (g) }\end{array}$ & $\begin{array}{l}\text { Pds du } \\
\text { jaune } \\
\text { (g) }\end{array}$ & $\begin{array}{c}\text { Jaune } \\
(\%)\end{array}$ & $\begin{array}{c}\text { Hauteur } \\
\text { albumen } \\
(0,1 \mathrm{~mm})\end{array}$ & $\begin{array}{c}\text { Épaisseur } \\
\text { de coquille } \\
(0,01 \mathrm{~mm})\end{array}$ & $\begin{array}{l}\text { Pds de } \\
\text { l'ouf } \\
\text { (g) }\end{array}$ & $\begin{array}{l}\text { Pds du } \\
\text { jaune } \\
\text { (g) }\end{array}$ & $\begin{array}{c}\text { Jaune } \\
(\%)\end{array}$ & $\begin{array}{c}\text { Hauteur } \\
\text { albumen } \\
(0,1 \mathrm{~mm})\end{array}$ & $\begin{array}{l}\text { Épaisseur } \\
\text { de coquille } \\
(0,01 \mathrm{~mm})\end{array}$ \\
\hline mars 1971 & 45,8 & 12,0 & 26,2 & 7,6 & 34,1 & 40,1 & 10,6 & 26,4 & 7,4 & 35,6 \\
\hline juin 1971 & 55,0 & 16,6 & 30,2 & - & - & 50,6 & 14,7 & 29,1 & - & - \\
\hline mars 1972 & 52,3 & 14,1 & 27,0 & 8,9 & 37,6 & 47,5 & 12,8 & 26,9 & 8,5 & 38,3 \\
\hline juin 1972 & 58,5 & 16,9 & 28,9 & 7,5 & 36,9 & 53,6 & 16,2 & 30,2 & 8,1 & 36,8 \\
\hline
\end{tabular}

\section{TABLEAU 7}

Analyse de variance de l'épaisseur moyenne de coquille par génotype et par famille de même mère (mars 197I)

\begin{tabular}{c|c|c}
\hline \hline Source de variation & Degrés de liberté & $\mathrm{F}$ \\
\hline & & \\
Génotype & 1 & $9,74^{* *}$ \\
Mère & 14 & 1,23 \\
Interaction & 14 & 1,32 \\
Résiduelle & 98 & - \\
\hline
\end{tabular}

** : Significatif au seuil 1 p. 100. 
On retrouve l'effet connu du gène $d w$ sur le poids de l'œuf et celui du jaune, mais on note au surplus qu'en début de ponte (mars I97I et I972), 1'épaisseur des coquilles des œufs des poules naines est sensiblement plus grande que pour les 우우 $D w$, alors que la différence disparait plus tard. Ceci est confirmé par l'analyse de variance du tableau 7, portant sur la période " mars I97I" (les données de mars 1972 étaient peu nombreuses.)

\section{TABLEAU 8}

Variation totale du poids de l'auf, du poids du jaune et de l'épaisseur de la coquille au cours de la série (I ${ }^{\mathbf{e r}}$-dernier œuf) pour des poules normales et naines de même origine

(nombres de séries indiqués entre parenthèses)

\begin{tabular}{|c|c|c|c|c|}
\hline \multirow{2}{*}{ Longueur de série } & \multicolumn{4}{|c|}{ Génotypes } \\
\hline & $D w$ & $d w$ & $D w$ & $d w$ \\
\hline & & Variation $d u$ & de l'œuf (g) & \\
\hline & & & ju & \\
\hline \multirow[t]{2}{*}{$\begin{array}{l}2 \text { et } 3 œ u f s \\
\text { plus de } 3\end{array}$} & $\begin{array}{l}(55)-1,25 \\
(40)-2,53\end{array}$ & $\begin{array}{l}(101)+0,21 \\
(54)+0,21\end{array}$ & $\begin{array}{l}(83)+1,62 \\
(29)+0,85\end{array}$ & $\begin{array}{r}(112)+0,95 \\
(9)+1,22\end{array}$ \\
\hline & \multicolumn{2}{|c|}{ mars 1972} & \multicolumn{2}{|c|}{ juin 1972} \\
\hline \multirow[t]{2}{*}{$\begin{array}{l}2 \text { et } 3 \text { œufs } \\
\text { plus de } 3\end{array}$} & $\begin{array}{l}(11)+0,84 \\
(11)-1,41\end{array}$ & $\begin{array}{l}(39)+1,81 \\
(14)+0,90\end{array}$ & $\begin{array}{l}(74)+1,82 \\
(26)-0,07\end{array}$ & $\begin{array}{r}(75)+1,59 \\
(7)-0,50\end{array}$ \\
\hline & \multicolumn{4}{|c|}{ Variation du poids du jaune $(\mathrm{g})$} \\
\hline \multirow[t]{2}{*}{$\begin{array}{l}2 \text { cufs } \\
3 \text { et } 4 \text { œufs }\end{array}$} & $\begin{array}{l}(9)-0,04 \\
(4)+0,02\end{array}$ & $\begin{array}{l}(18)+0,10 \\
(15)+0,14\end{array}$ & $\begin{array}{l}(10)+0,78 \\
(5)+0,84\end{array}$ & $\begin{array}{r}(21)+0,32 \\
(3)+0,60\end{array}$ \\
\hline & \multicolumn{2}{|c|}{ mars 1972} & & \\
\hline \multirow[t]{3}{*}{$\begin{array}{l}2 \text { œufs } \\
\text { plus de } 2 \text { œufs }\end{array}$} & $\begin{array}{l}(8)+0,25 \\
(14)-0,33\end{array}$ & $\begin{array}{l}(27)+0,33 \\
(26)+0,51\end{array}$ & & \\
\hline & \multicolumn{4}{|c|}{ Variation de l'épaisseur de la coquille $(0,01 \mathrm{~mm})$} \\
\hline & \multicolumn{2}{|c|}{ mars 1971} & & \\
\hline \multirow[t]{2}{*}{$\begin{array}{l}2 \text { et } 3 \text { œufs } \\
\text { plus de } 3\end{array}$} & $\begin{array}{l}(49)-0,93 \\
(20)-0,87\end{array}$ & $\begin{array}{l}(54)-1,94 \\
(24)-2,67\end{array}$ & & \\
\hline & \multicolumn{2}{|c|}{ mars 1972} & \multicolumn{2}{|c|}{ juin 1972} \\
\hline $\begin{array}{l}2 \text { et } 3 \text { œufs } \\
\text { plus de } 3\end{array}$ & $\begin{array}{l}(11)-1,70 \\
(11)+1,27\end{array}$ & $\begin{array}{l}(39)-1,91 \\
(14)-0,14\end{array}$ & $\begin{array}{l}(73)-1,81 \\
(26)-1,16\end{array}$ & $\begin{array}{r}(75)-1,53 \\
(7)-1,57\end{array}$ \\
\hline
\end{tabular}


TABLEAU 9

Analyse de variance de la variation de poids de l'aut dans la série de ponte

\begin{tabular}{|c|c|c|c|c|}
\hline \multirow{2}{*}{$\begin{array}{c}\text { Source } \\
\text { de variation }\end{array}$} & \multicolumn{2}{|c|}{ Mars 1971} & \multicolumn{2}{|c|}{ Juin 1971} \\
\hline & $\begin{array}{l}\text { degrés } \\
\text { de liberté }\end{array}$ & F & $\begin{array}{l}\text { degrés } \\
\text { de liberté }\end{array}$ & $\mathrm{F}$ \\
\hline Génotype & 1 & $6,50^{* *}$ & 1 & 0,03 \\
\hline Série $\ldots \ldots$ & 3 & 1,56 & 3 & 0,12 \\
\hline Interaction & 3 & 0,88 & 3 & 0,29 \\
\hline Résiduelle ... & 242 & & 225 & \\
\hline
\end{tabular}

** : Significatif au seuil 1 p. 100.

Pour la hauteur de l'albumen, au contraire, elle ne présente pas de différence entre $D w$ et $d w$ en mars, alors qu'en juin 1972, où sa mesure a pu être faite, on retrouve une valeur plus élevée chez les poules naines que chez les normales, confirmant des résultats antérieurs obtenus au même âge (MÉRAT, I972).

Le tableau 8 donne la variation totale du poids de l'œuf (différence moyenne entre premier et dernier œuf), du poids du jaune et de l'épaisseur de la coquille du début à la fin de séries de tailles différentes. Sur ces caractères, une analyse de variance a été faite, avec les facteurs contrôlés " génotype " ( $D w$ ou $d w)$ et " taille de série " en r97I (les données de I972, mars surtout, étant moins nombreuses) : nous ne la présentons que pour le poids des œufs (tab1. 9), les analyses sur les deux autres critères ne faisant apparaître aucun effet significatif.

On voit que, du moins en mars, il existe un effet " génotype " hautement significatif, le poids de l'œuf diminuant davantage du début à la fin de la série chez les poules $d w$. La tendance est de même sens dans les deux années. Quoique la différence entre génotypes ne soit pas significative, la variation du poids du jaune est parallèle à celle du poids de 1'œuf entier. Une tendance semble également exister à une augmentation plus grande de l'épaisseur de la coquille pour les poules naines en mars.

\section{DISCUSSION E'T CONCLUSIONS}

Le décalage plus grand de l'heure de ponte des œufs consécutifs d'une même série chez les poules naines $d w$ peut provenir d'un écart de temps plus grand entre ovulations successives, reflétant une différence d'intensité de ponte avec les normales. Cette intensité, sur la moyenne des périodes qui nous intéressent, était respectivement 73,9 p. roo et 6r,7 p. Ioo chez les normales et les naines en I97r, les valeurs correspondantes en 1972 étant 74,3 èt 6r,7 p. Ioo. 
De fait, l'intensité de la vitellogenèse ayant été trouvée inférieure chez les poules naines comparées aux normales (JAAP et MOHAMMADIAN, I969), une maturation plus tardive des follicules peut retarder l'ovulation chez les premières. La diminution plus grande du poids de l'œuf ou du jaune des naines au cours des séries s'accorde aussi avec cette hypothèse. Cependant, il ne paraît pas certain que ceci soit la seule cause du décalage plus grand entre ovipositions chez les naines, car en juin, où la différence de celles-ci avec les normales est bien moins importante qu'en mars (sauf pour l'heure de ponte des œufs isolés), la longueur de leurs séries est plus réduite que pour les poules $D w(2,60$ et r,83 en mars et en juin I97r, les valeurs correspondantes pour les $q 9$ Dw étant 2,76 et 2,I5). D'autre part, les œufs uniques (séries de I) sont pondus plus tard par les naines, et comme ils constituent un début de série, il n'est pas sûr qu'ils correspondent à des retards d'ovulation. On peut donc se demander s'il n'intervient pas aussi une durée plus grande de séjour de l'œuf dans l'utérus chez les poules naines, en mars du moins.

En ce qui concerne l'effet de la saison (mars ou juin) sur l'heure de ponte et son décalage dans la série, l'importance dans l'ensemble plus grande de ce dernier en juin est parallèle à la baisse d'intensité de ponte à cette période, reflétant une évolution interne de l'oiseau au cours du cycle de ponte, ou l'effet de facteurs saisonniers.

Parmi ces derniers, NordSTrom (I973) a montré qu'une température plus élevée augmente la durée de séjour de l'œuf dans l'utérus. D'autre part, l'influence de ces facteurs, internes ou externes, s'exerce probablement aussi dans le sens d'une diminution de l'intensité de la vitellogenèse de mars à juin, à en juger par la décroissance plus marquée, à cette époque, du poids du jaune et de l'œuf entier dans la série.

Quant à l'effet favorable du gène $d r$ : sur l'épaisseur de la coquille en début de ponte, non retrouvé plus tard d'après nos observations présentes et antérieures et celles d'autres auteurs, il pourrait correspondre simplement à la maturité sexuelle d'environ 2 semaines plus tardive des naines (MÉRAT, I972). Inversement, la consistance plus grande de l'albumen pour ce génotype trouvée ici en juin, ainsi que précédemment au même stade (MÉRAT, I972) semble n'apparaître que progressivement et ne pas exister en début de ponte.

Reçu pour publication en janvier 1975.

\title{
SUMMARY
}

\author{
STUDY OF A SEX LINKED DWARFING GENE IN THE FOWL : \\ OVIPOSITION TIME AND CHARACTERISTICS OF THE SUCCESSIVE EGGS \\ IN LAYING SEQUENCES
}

The approximate oviposition time was recorded for successive eggs in laying sequences (or "clutches") of varying size, at the beginning of laying (March I97I), then after 4 to 5 months production (June 197I) for dwarf $(d w)$ and normal-sized $(D w)$ hens. Eggs from one-egg clutches are laid later in the day for dwarf hens. This is not the case for the first egg of longer sequences; but the lag in the oviposition time between successive eggs in these sequences is more important in $d w$ hens, especially in March. On the other hand, this lag is, for the two genotypes, higher in June than in march.

Egg weight, yolk weight, albumen height and shell thickness were also measured, according to the place of the egg in the laying sequence, for the same $D w$ and $d w$ hens in March and June 
1971, and for others in March and June 1972. On the whole, egg and yolk weight decrease more and shell thickness seems to increase more, from the beginning to the end of a sequence, for dwarf hens. This confirms previous observations.

On the other hand, shell thickness on the average is higher for dwarfs at the beginning of production, this possibly being due entirely to their later sexual maturity.

\section{RÉFÉRENCES BIBLIOGRAPHIQUES}

Fraps R. M., I955. In : J. Hammond Progress in the physiology of farm animal, 2, Butterworths, London. Hutr F. B., 1959. Sex-linked dwarfism in the fowl. J. Hered., 50, 209-221.

JaAp R. G., Mohammadian M., 1969. Sex-linked dwarfism and egg production of broiler dams. Poult. Sci., 48, 344-346.

Merat P., r972. Quelques effets du gène dw sur la ponte et sur la qualité des œufs. Ann. Génét. Sél. anim., 4, 2 I7-223.

Nordstrom J. O., I973. Duration of egg formation in chickens during heat stress. Poult. Sci., 52, I687-I690.

Snedecor G. W., Cochran W. G., 1969. Statistical methods., 6e éd, Iowa State Univ. Press, Ames. 